\title{
Small Vessel Disease, a Marker of Brain Health: What the Radiologist Needs to Know
}

\author{
(D) A. Mahammedi, (D) L.L. Wang, (D)B.J. Williamson, (DP. Khatri, (D) B. Kissela, (D).P. Sawyer, (D). Shatz, (D) V. Khandwala, and \\ (iD) A. Vagal \\ O- $\equiv$
}

\begin{abstract}
SUMMARY: Small vessel disease, a disorder of cerebral microvessels, is an expanding epidemic and a common cause of stroke and dementia. Despite being almost ubiquitous in brain imaging, the clinicoradiologic association of small vessel disease is weak, and the underlying pathogenesis is poorly understood. The STandards for Reportlng Vascular changes on nEuroimaging (STRIVE) criteria have standardized the nomenclature. These include white matter hyperintensities of presumed vascular origin, recent small subcortical infarcts, lacunes of presumed vascular origin, prominent perivascular spaces, cerebral microbleeds, superficial siderosis, cortical microinfarcts, and brain atrophy. Recently, the rigid categories among cognitive impairment, vascular dementia, stroke, and small vessel disease have become outdated, with a greater emphasis on brain health. Conventional and advanced small vessel disease imaging markers allow a comprehensive assessment of global brain heath. In this review, we discuss the pathophysiology of small vessel disease neuroimaging nomenclature by means of the STRIVE criteria, clinical implications, the role of advanced imaging, and future directions.
\end{abstract}

ABBREVIATIONS: BOLD = blood oxygen level-dependent; CAA = cerebral amyloid angiopathy (SVD type 2); CMB = cerebral microbleeds; $\mathrm{CSS}=$ cortical superficial siderosis; $\mathrm{HA}=$ hypertensive arteriolosclerosis (SVD type 1); ICH = intracerebral hemorrhage; PVS $=$ prominent perivascular spaces; STRIVE = STandards for ReportIng Vascular changes on nEuroimaging; SVD = small vessel disease; WMH = white matter hyperintensities

S mall vessel disease (SVD) is an umbrella term of disease processes affecting the small arteries, arterioles, venules, and capillaries of the brain. The small vessel injuries cause lesions that can be detected on pathologic examination and that are now increasingly being recognized on brain imaging. ${ }^{1,2}$ Although SVD can be asymptomatic, it commonly coexists with neurodegenerative diseases and can exacerbate cognitive impairment, increase the risk of stroke, and worsen outcome after stroke. ${ }^{3-6}$ In fact, SVD is an enormous health burden, causing about $25 \%$ of ischemic strokes and most hemorrhagic strokes, is the most common cause of vascular dementia, and contributes to about half of dementias worldwide. ${ }^{3,5-8}$ As the rigid boundaries among vascular dementia, stroke, and SVD fade, the common theme now is brain health. ${ }^{9,10}$ The spectrum of SVD includes white matter hyperintensities (WMH) of presumed vascular origin, recent small subcortical infarcts, lacunes, microbleeds, superficial siderosis,

Received April 18, 2021; accepted after revision July 5.

From the Departments of Neuroradiology (A.M., L.L.W., B.J.W., V.K., A.V.) and Neurology (P.K., B.K., R.P.S., R.S.), University of Cincinnati Medical Center, Cincinnati, Ohio.

Please address correspondence to Abdelkader Mahammedi, MD, Department of Neuroradiology, University of Cincinnati Medical Center, 234 Goodman St, Cincinnati, OH; e-mail: abdelkm2@gmail.com

- Indicates open access to non-subscribers at www.ajnr.org

Indicates article with online supplemental data.

http://dx.doi.org/10.3174/ajnr.A7302 prominent perivascular spaces, microinfarcts, and brain atrophy. ${ }^{11,12}$ There have been competing systems in the past regarding the neuroimaging standards for classification of SVD. However, due to the substantial variation in how studies have defined SVD on conventional MR imaging, ${ }^{13,14}$ a most recent international effort to establish clinical and research standards to facilitate a more consistent approach in describing SVD neuroimaging was proposed by the STandards for ReportIng Vascular changes on nEuroimaging (STRIVE). ${ }^{11}$

There are different types and etiopathogenic classifications of SVD, with a proposed classification (Table 1). ${ }^{1}$ Type 1 , hypertensive arteriolosclerosis (HA), and type 2, cerebral amyloid angiopathy (CAA) are, by far, the most common sporadic types of SVD in older adults and the most radiologically well-established entities. ${ }^{15}$ Although most SVD is sporadic, likely related to hypertension or other vascular risk factors, a few forms are related to rare genetic diseases (for example, CADASIL; cerebral autosomal recessive arteriopathy with subcortical infarcts and leukoencephalopathy; Fabry disease; Hereditary Endotheliopathy with Retinopathy, Nephropathy, and Stroke syndrome; and COL4AI), of which CADASIL is the most common (Table 1$)^{5}$

In this review, we discuss the underlying mechanisms and pathophysiology of SVD neuroimaging nomenclature using the STRIVE criteria, clinical implications, the role of advanced imaging, and future directions. Because SVD is almost ubiquitous in 


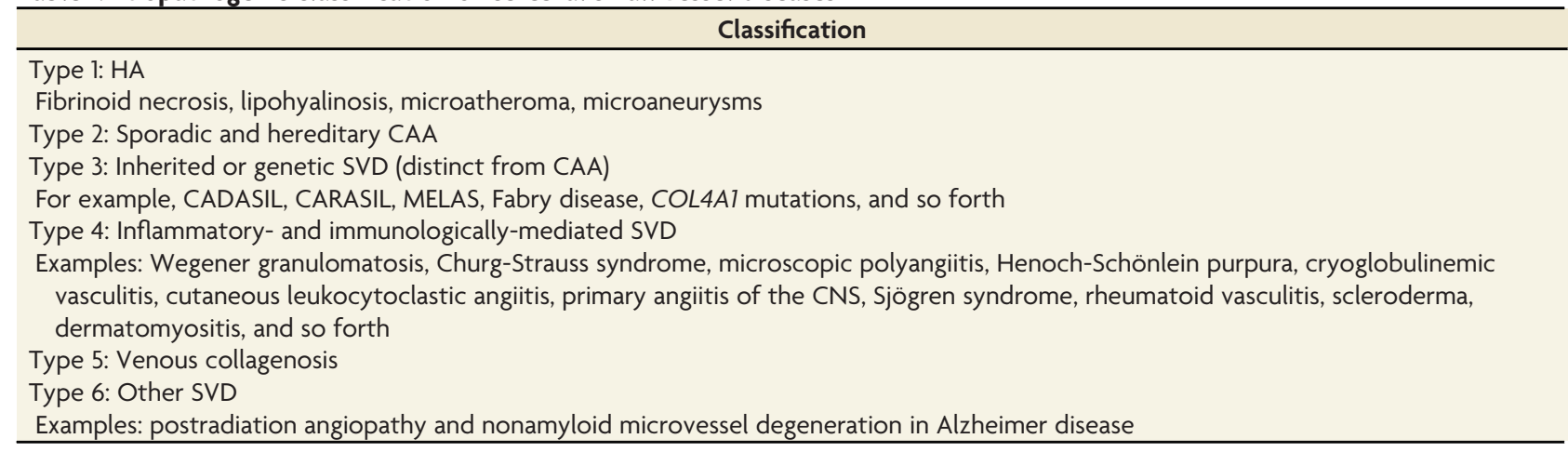

Note:-MELAS indicates Mitochondrial Encephalopathy, Lactic Acidosis, and Stroke-like episodes; CARASIL, cerebral autosomal recessive arteriopathy with subcortical infarcts and leukoencephalopathy.

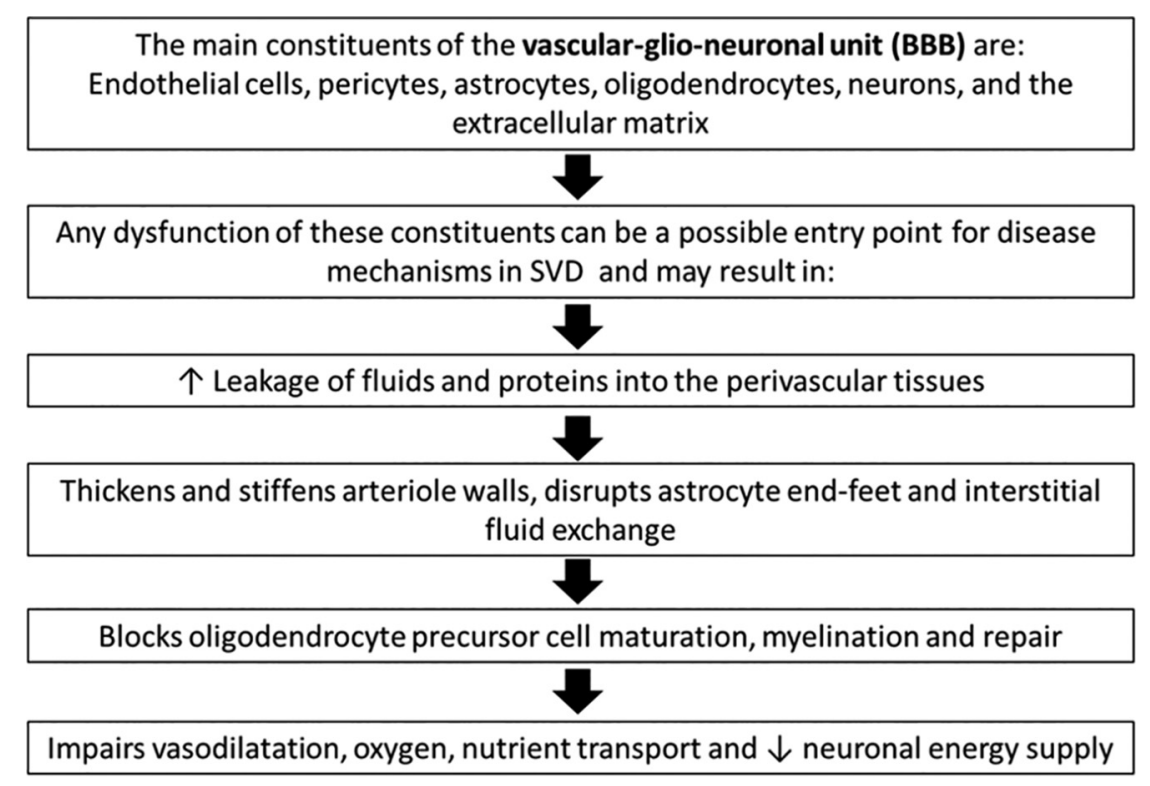

FIG 1. Possible entry points in the pathophysiology of SVD. The order of these events has not been determined.

adult brain imaging, it is important for radiologists to be familiar with the imaging spectrum, nomenclature, and clinical relevance.

\section{Pathophysiology and Epidemiology of SVD}

The exact pathogenesis of SVD is incompletely understood, but the most common abnormalities are diffuse arteriolosclerosis, lipohyalinosis, and fibrinoid necrosis of small arterioles. ${ }^{2,16-18}$ Additionally, embolism or atheroma and other vascular risk factors (diabetes mellitus, hypertension, and hypercholesterolemia) are also believed to play a role. ${ }^{2}$ Fisher $^{16}$ suggested that symptomatic lacunar infarcts were caused by atherosclerosis or embolism in the larger arterioles, whereas the silent infarcts were likely attributable to lipohyalinosis or fibrinoid necrosis in the smaller arterioles. ${ }^{2}$ Lacunar infarcts were traditionally considered to be mainly caused by lipohyalinosis of small perforating arteries. However, atherosclerosis in the parental artery can produce a similar single lacunar infarct by blocking the orifice of branching arteries. ${ }^{19}$ A systematic review of Asian studies reported that parent artery atherosclerosis resulted in $20 \%$ of single lacunar infarcts. There are four possible mechanisms for lacunar ischemic stroke that have been proposed: atheroma of parent arteries (usually MCA) or perforating arterioles, embolism from the heart or carotid arteries, and intrinsic SVD (lipohyalinosis or fibrinoid necrosis). ${ }^{19,20}$

Although hypertension is a key risk factor, many patients with SVD are not hypertensive. ${ }^{2,21-24}$ Recent studies suggest more complex mechanisms other than arteriolosclerosis that can cause capillary wall dysfunction. The deposition of lipids in arteriolar walls (microatheroma) can damage the endothelium (Fig 1), ${ }^{2,5}$ leading to leakage of plasma proteins and inflammatory cells into the perivascular tissues. ${ }^{5,25}$ The BBB dysfunction involving the neurogliovascular unit can result in vessel stiffening, inflammation, myelin damage, and secondary neurodegeneration. ${ }^{2,5,26-28}$ The neurogliovascular unit has multiple possible entry points for disease mechanisms (ie, endothelial cells, pericytes, astrocytes, oligodendrocytes, neurons, and the extracellular matrix). At a cellular level, the disruption of normal astrocyte function decreases neuronal energy supply, resulting in BBB leakage. ${ }^{2,25,29}$ Although the exact order of these events is not well-established, these cellular changes of endothelial failure and inflammation are more dynamic and widespread than previously thought. ${ }^{2,5}$ It was also hypothesized that in rodents, prominent perivascular spaces can be associated with a blockage of the drainage of interstitial fluid from increased vessel stiffness and increased arteriolar pulsatility such as with arterial hypertension, which results in fluid stagnation in the perivascular spaces and thus decreased interstitial fluid flushing. ${ }^{5,30}$ This result may impede the clearance of metabolites (including $\beta$-amyloid and other proteins) from tissues. Alzheimer disease, cerebral amyloid angiopathy, and several monogenic small vessel diseases are thus being considered as protein elimination failure angiopathies., ${ }^{5,30}$

Although there is significant variation in the epidemiology of SVD markers, the prevalence of these markers is associated with 


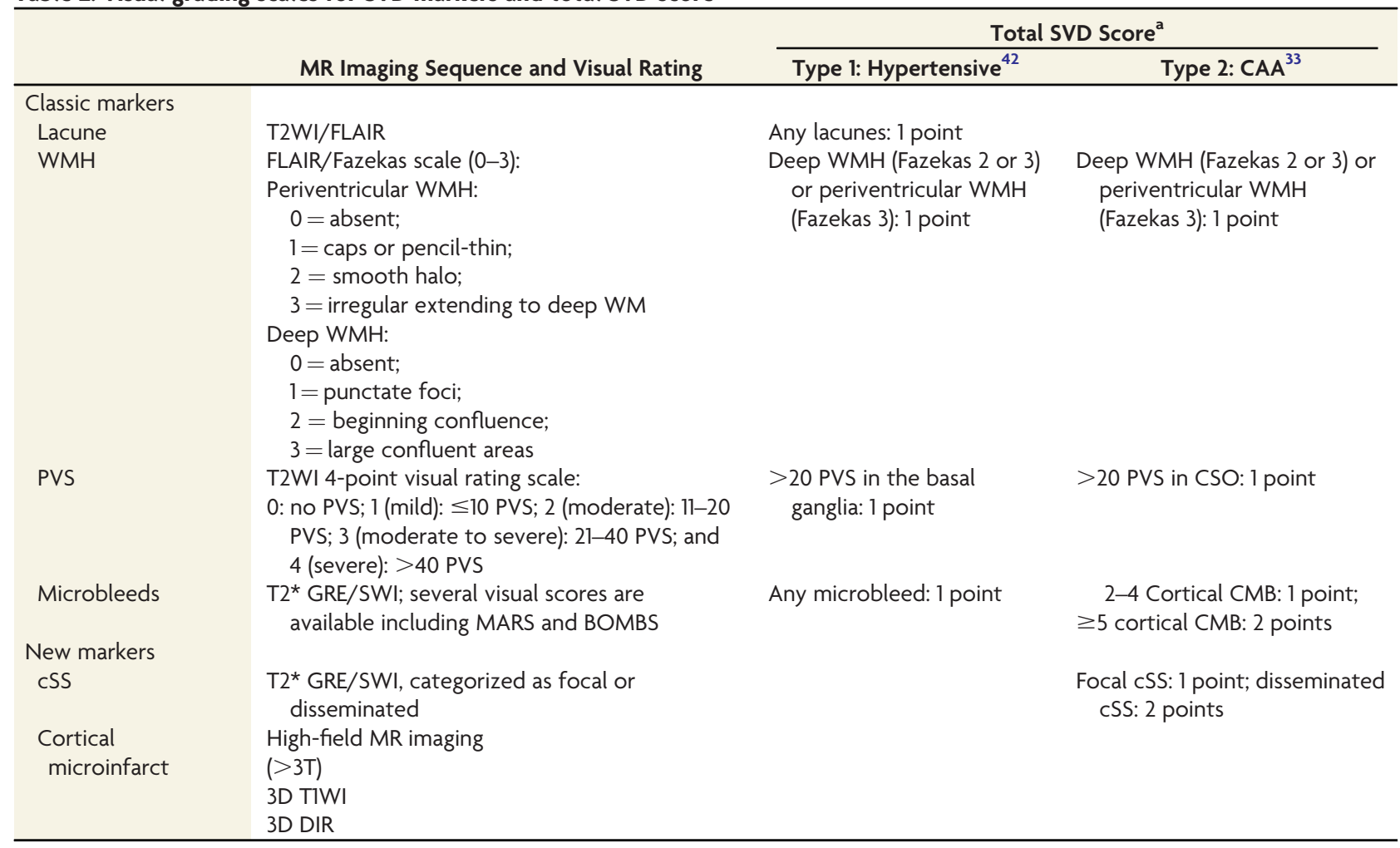

Note:-GRE indicates gradient recalled-echo; MARS, Microbleed Anatomical Rating Scale; BOMBS, Brain Observer MicroBleed Scales; CSO, centrum semiovale; cSS, cortical superficial siderosis; BG, basal ganglia; DIR, double inversion recovery.

${ }^{a}$ Type 1: Hypertensive; type 2: CAA.

greater age. The association between white matter hyperintensities, lacunes, prominent perivascular spaces (PVS), and cerebral microbleeds (CMB) and aging has been well-documented. ${ }^{31}$ Other demographic factors such as sex and ethnicity/race can also explain the variability in SVD, though the exact mechanism is poorly understood. ${ }^{31}$ It was reported that although Blacks and Asians have a high burden of SVD compared with Whites, Blacks had a lower prevalence of $\mathrm{WMH}$ but a higher prevalence of severe $\mathrm{WMH}^{32}$ Several population-based studies have characterized SVD in the "healthy population" and explored the risk factors for SVD. ${ }^{32}$

\section{Neuroimaging of SVD}

Nomenclature and Definitions. Radiologists are well-versed with the variations in the radiology reports describing SVD, for example, $\mathrm{WMH}$, cerebral white matter disease, leukoaraiosis, and agerelated white matter disease. The STRIVE guidelines and standards were developed to address this variability (Online Supplemental Data). ${ }^{11}$ The recommendations were initially applied to research studies with the potential goal of widespread clinical use to standardize image interpretation, acquisition, and reporting. The classic MR imaging markers of SVD include recent small subcortical infarctions, lacunes of presumed vascular origin, WMH of presumed vascular origin, PVS, and CMB. Two additional MR imaging markers of SVD that occur with high prevalence in CAA are cortical superficial siderosis (cSS) and cortical microinfarcts (Table 2). ${ }^{33}$

Recent Small Subcortical Infarcts. According to the STRIVE criteria, the term "recent small subcortical infarcts" refers to a recent infarct occurring in the territory of a perforating arteriole with symptoms or imaging features that suggest a timeline of a previous few weeks. However, in most patients, these lesions can appear without apparent clinical symptoms (silent infarcts) (Online Supplemental Data). ${ }^{11}$ The term "recent" is preferred instead of "acute" because it includes both the hyperacute stage and the first few weeks of the lesion. ${ }^{11}$ These lesions typically measure $\leq 20 \mathrm{~mm}$ in their maximum diameter in the axial plane, though in the coronal plane, they can appear slightly larger and elongated, reflecting the territory of the occluded perforating arteriole (Fig 2A). They are best identified with hyperintense signal on DWI, hypointense signal on an ADC map, and hyperintense signal on T2-weighted and FLAIR sequences. The most common fate of these lesions on longterm follow-up is cavitation to become a lacune (in $28 \%-94 \%$, depending on the duration of follow-up), turning into a nonspecific focus of T2/FLAIR hyperintensity, or eventually completely disappearing. ${ }^{2,8}$ Furthermore, STRIVE proposes that lesions in the basal ganglia and internal capsule of $>20 \mathrm{~mm}$ are a different subtype and, therefore, should be classified as striatocapsular infarcts instead of small subcortical infarcts. Similarly, infarcts of the anterior choroidal artery are etiologically and anatomically distinct.

Lacunes of Presumed Vascular Origin. The STRIVE guidelines define lacunes of presumed vascular origin as round or ovoid, subcortical, fluid-filled cavities (with signal similar to that of CSF in all sequences), measuring between 3 and $15 \mathrm{~mm}$ in diameter, consistent with a previous, small subcortical infarct or hemorrhage located in deep gray and white matter and in a territory of perforating arteriole (Fig $2 B$ and Online Supplemental Data). These lesions 


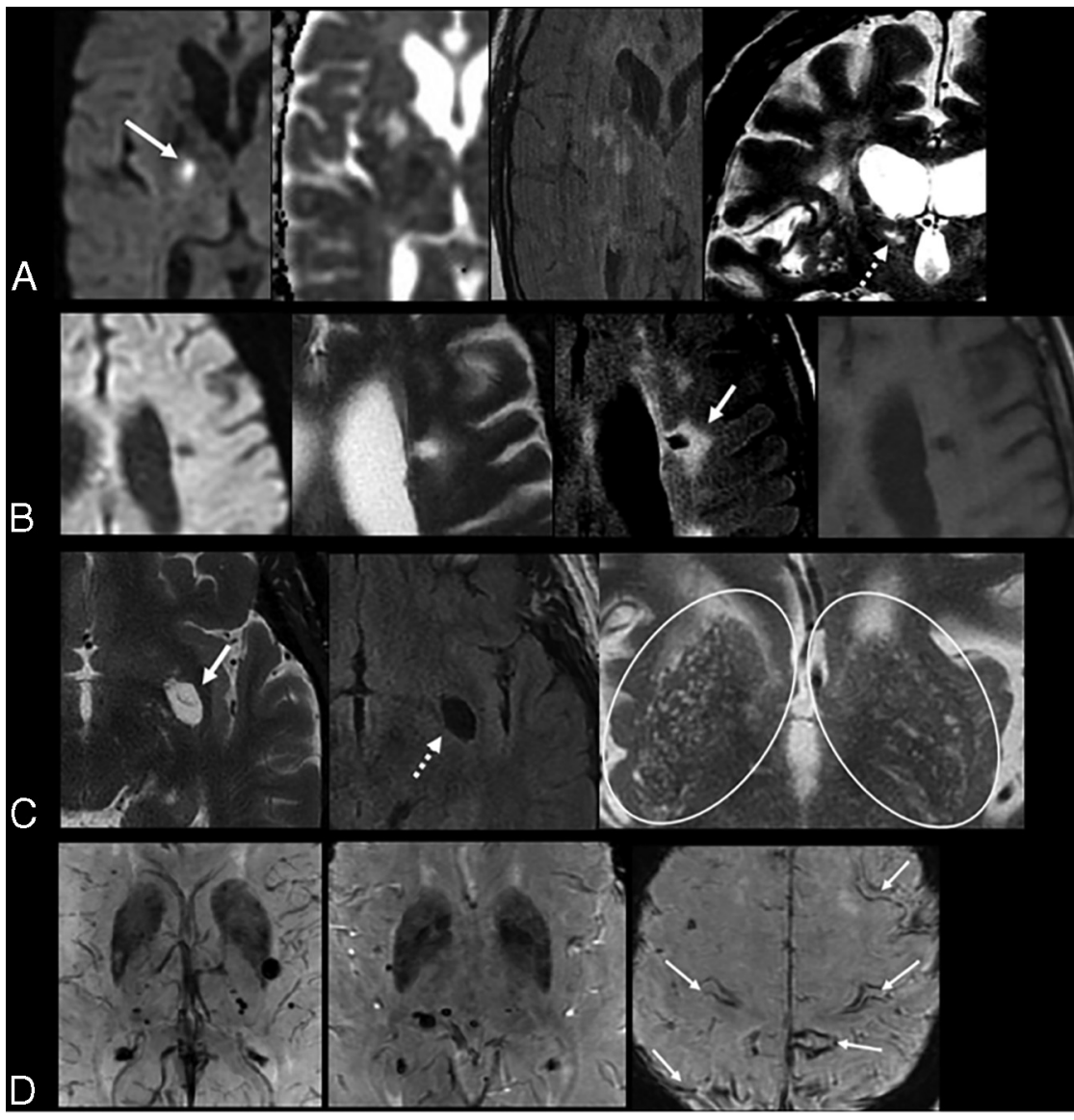

FIG 2. A, Recent subcortical infarct in the right lentiform nucleus in a 59-year-old woman with a history of hypertension. From left to right, axial DWI, ADC map, FLAIR, and coronal T2WI show restricted diffusion (solid white arrow, A). On coronal T2WI, the elongated morphology of the acute infarct in the craniocaudal axis (dashed white arrow, $A$ ) is related to the territory of a perforating artery. $B$, A lacune of presumed vascular origin in a 67-year-old man with a history of dementia. From left to right, axial DWI, T2WI, FLAIR, and TTWI show a remote lacunar infarct in the left frontal corona radiata, which demonstrates $\mathrm{T} 2$ hyperintensity with a peripheral rim of gliosis, best seen on FLAIR image (solid white arrow, B). C, Prominent perivascular spaces in a 63-year-old man with a history of dementia. A prominent perivascular space is noted in the left insular region with a centrally traversing vessel (solid white arrow, C), without peripheral gliosis on the FLAIR image (dashed arrow, C). Additionally, there are $>20$ dilated perivascular spaces in the bilateral basal ganglia on axial T2WI (circles, C). D, Cerebral microbleeds with cSS in an 87-year-old man with a history of CAA who presented with a worsening mental status. Axial SWI demonstrate multiple foci of susceptibility artifacts predominantly involving the basal ganglia and thalami, consistent with microbleeds. Additional areas of scattered cortical subarachnoid hemorrhagic staining (arrows, $D)$ indicate cortical superficial siderosis.

typically demonstrate a surrounding gliotic rim of T2 FLAIR hyperintensity (Figs 3 and 4), which can be a useful feature when present. A peripheral gliotic rim can also surround perivascular spaces when they pass through an area of white matter hyperintensity. Although lacunes are commonly seen in older patients without symptoms, they are associated with an increased risk of stroke, dementia, and gait impairment. ${ }^{5,11}$

WMH of Presumed Vascular Origin. WMH of presumed vascular origin are characterized by hyperintense lesions on T2 FLAIR and decreased attenuation on CT in the periventricular/deep cerebral white matter, subcortical gray matter, basal ganglia, and brainstem. Although the Fazekas visual rating scale (Fig 3 ) is the most commonly used method to assess the burden of WMH, there is a lack of a rigorous method to accurately quantify the WMH burden. ${ }^{34}$ Furthermore, a fronto-occipital gradient was used in few studies to describe the difference in the severity of WMH between the frontal and occipital lobes. ${ }^{33,35}$ It was suggested that patients with obvious occipital-dominant WMH were more likely to develop complications from SVD such as lobar intracranial hemorrhage than healthy controls. ${ }^{35}$ The severity of WMH is strongly associated with cerebrovascular disease, vascular risk factors, gait disturbance, cognitive symptoms, and poststroke functional outcomes. ${ }^{3,11}$

Perivascular Spaces, Also Called Virchow-Robin Spaces. Perivascular spaces are extensions of the extracerebral fluid space that are covered by the pia mater, which surrounds cerebral vessels from the brain surface into and through the brain parenchyma. These are commonly microscopic and not visible on conventional neuroimaging, but when they become prominent and numerous, they are considered a marker of SVD. PVS have been shown to be associated with other SVD markers such as WMH and lacunes, but not atrophy. ${ }^{11}$ PVS have a signal intensity similar to that of CSF in all sequences and appear round or ovoid with a diameter of $<3 \mathrm{~mm}$ but can appear more elongated when imaged parallel to the course of the penetrating vessel. They are typically located in the inferior basal ganglia, centrum semiovale, and midbrain and do not have a surrounding gliotic rim (in contrast with lacunes) (Fig 2C and Online Supplemental Data). Although lacunes and PVS can have similar MR imaging features, pathologic studies have demonstrated that lesions that are $<3 \mathrm{~mm}$ in diameter are more likely to be perivascular spaces than lacunes. ${ }^{11}$ A traversing vessel can sometimes be seen in the center of a perivascular space when imaged at high resolution, another differentiator of PVS versus lacunes (Fig 2C). The severity of PVS is typically assessed on axial T2-weighted images using a validated 4-point visual rating scale based on the total number of PVS (0 indicating no PVS; 1 [mild], $\leq 10$ PVS; 2 [moderate], 11-20 PVS; 3 [moderate to severe], 21-40 PVS; and 4 [severe], $>40$ PVS) in the basal ganglia and centrum semiovale (Table 


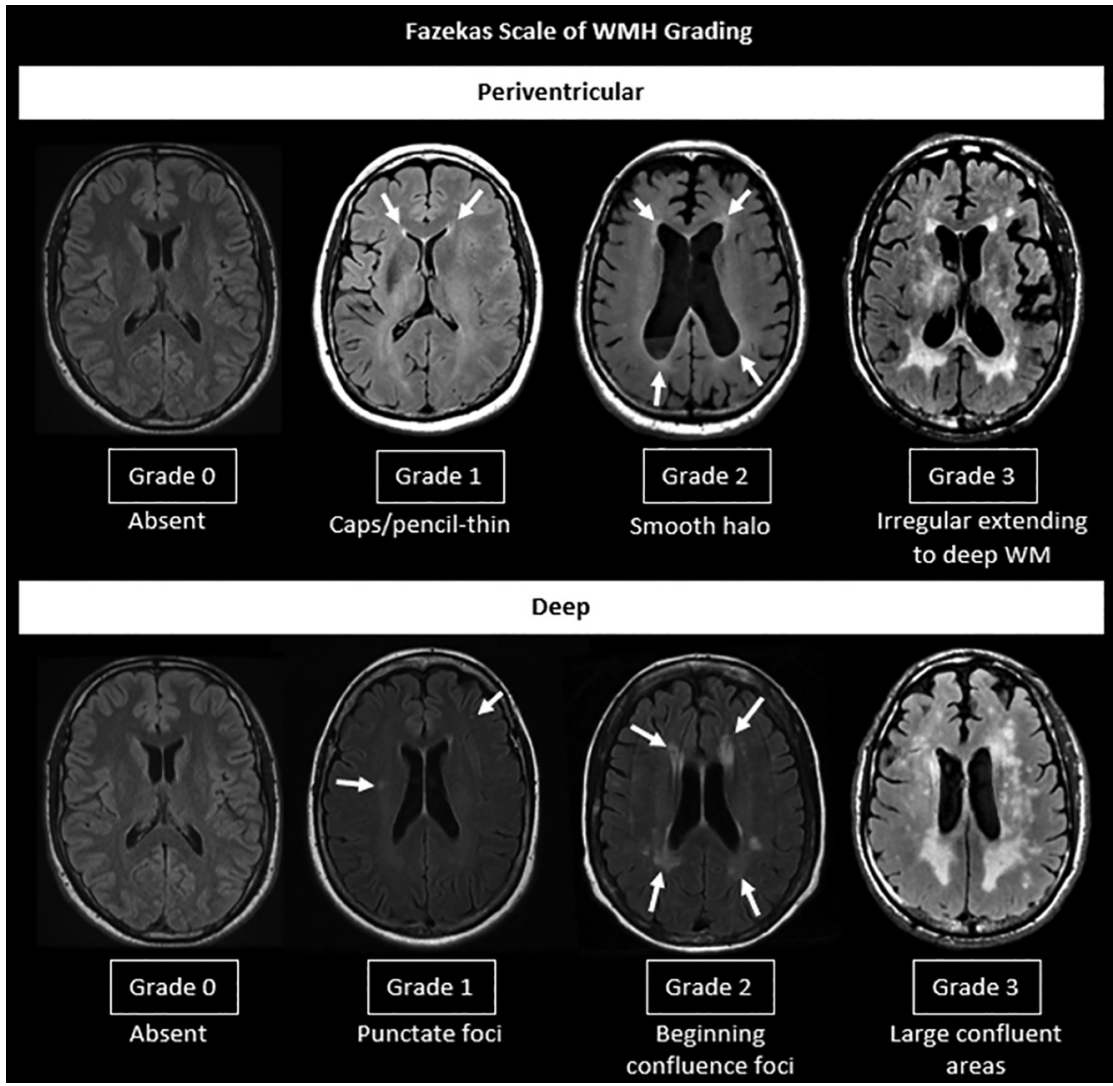

FIG 3. Fazekas scale of WMH grading.
2). ${ }^{33}$ The topography of PVS can be a helpful characteristic of the underlying SVD type: PVS are likely associated with arteriolosclerosis when located in the basal ganglia, and they are more likely to be related to CAA when located in the centrum semiovale (Fig 4). ${ }^{10}$ However, the clinical significance of PVS remains unclear; a few studies have reported that PVS can be associated with an increased risk of dementia if located in the basal ganglia and white matter. ${ }^{5,10,36}$

CMB. CMB are small round or ovoid lesions ( $\leq 10 \mathrm{~mm}$ in diameter) of marked hypointensity with associated blooming on $\mathrm{T} 2$ gradient-echo $\left(\mathrm{T} 2{ }^{*}\right)$ or other sequences that are sensitive to susceptibility effects, especially SWI (Fig 2D and Online Supplemental Data). CMB correspond to hemosiderin-laden macrophages in perivascular tissue, consistent with vascular leakage of blood cells. ${ }^{5}$ Several visual scores are available, including the Microbleed Anatomical Rating Scale and the Brain Observer MicroBleed Scales, which

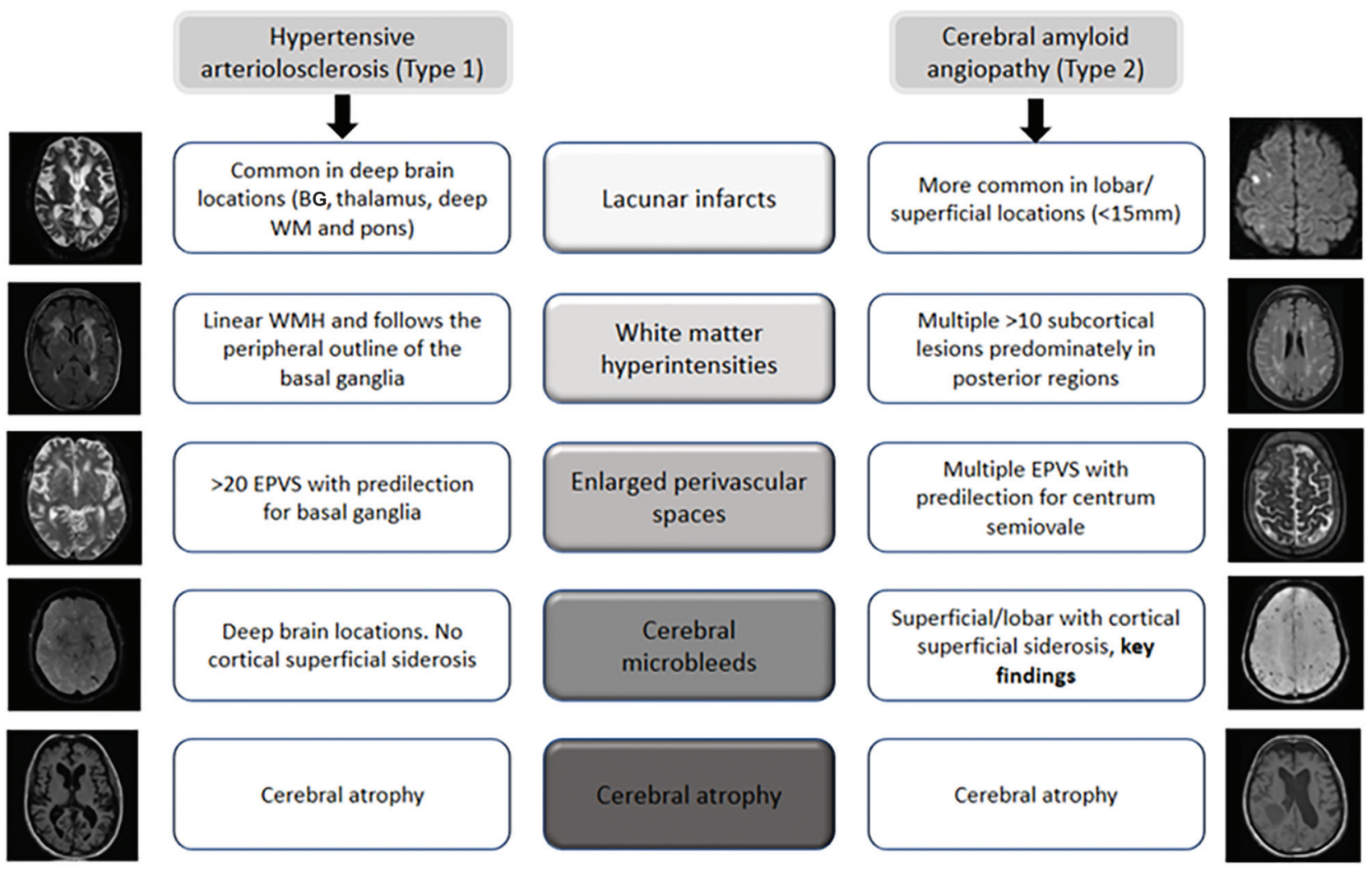

FIG 4. SVD MR imaging markers of the two most common etiopathogenic types of cerebral SVD in older adults, HA and CAA. BG indicates basal ganglia; EPVS: enlarged prominent perivascular spaces. 


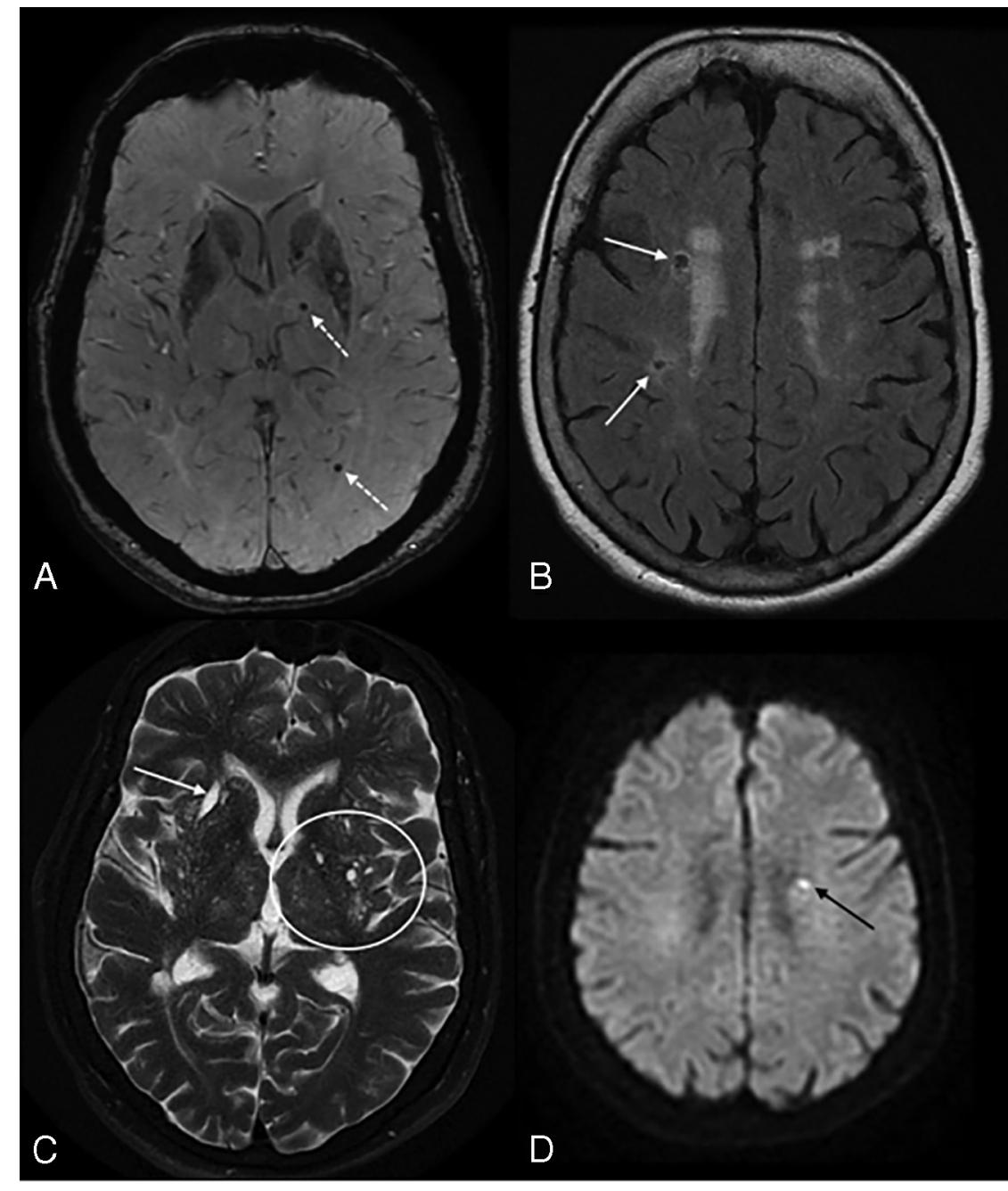

FIG 5. High SVD burden in a 55-year-old man with a history of arterial hypertension. Note multiple microbleeds in the left thalamus and occipital lobe (dotted arrows) on SWI (A), remote lacunar infarcts in the right centrum semiovale (white arrows, B), and WMH Fazekas 2 on FLAIR (B). More than 20 dilated perivascular spaces are seen on axial T2WI (circle, C) and remote lacunar infarct in right lentiform nucleus (white arrow, C). The total SVD score is 4. The patient developed an acute lacunar infarct (black arrow) on DWI (D) 10 months later.

have reasonable intrarater and interrater reliability for the presence of definite microbleeds. ${ }^{37,38}$ In fact, a few recent publications have proposed a novel automatic method to detect $\mathrm{CMB}$ from $\mathrm{MR}$ images by exploiting the $3 \mathrm{D}$ convolutional neural network. ${ }^{39} \mathrm{CMB}$ are associated with an increased risk of cognitive decline and ischemic and hemorrhagic stroke, though the risk of dementia appears higher in patients with $\mathrm{CMB}$ restricted to a superficial/lobar location compared with those with deep involvement. ${ }^{5,10}$ Two other hemorrhagic manifestations of SVD include cSS and spontaneous intracerebral hemorrhage (in contrast to secondary hemorrhage due to other causes such as trauma and vascular malformations).

\section{Total SVD Score}

Although the neuroimaging markers have typical characteristics, in practice, it is difficult to distinguish lacunes, PVS, and WMH of presumed vascular origin because they are often closely related. A few studies have suggested combining these MR imaging markers and using a more comprehensive approach to assess total SVD burden. ${ }^{33,40}$ Total SVD score is a simple and pragmatic way of assessing overall brain health and has been shown to be a prognostic indicator of cognitive decline and recurrent stroke (Fig 5). The total burden of lesions in specific anatomic locations can also be used for lesion-symptom associations and has been shown to be associated with cognitive impairment and gait and mood disorders. $^{5,40,41}$ For instance, a total SVD score in CAA was proposed by Charidimou et $\mathrm{al}^{33}$ and can potentially provide a more practical framework to better evaluate the effect of CAArelated brain damage on clinical outcomes. A similar total MR imaging SVD burden approach was proposed in HA by Klarenbeek et al. ${ }^{42}$ The imaging rating points of total SVD burden are outlined in Table 2.

\section{Imaging in $\mathbf{H A}$ versus CAA}

HA (type 1) and CAA (type 2) are the most common sporadic SVD types in older adults (Fig 4 and Table 2) with intrinsically different pathophysiology, clinical significance, and prognosis. ${ }^{15}$ HA predominantly affects small perforating end arteries of the deep gray nuclei and deep white matter, whereas CAA results from $\beta$-amyloid deposition within the cortical and leptomeningeal arteries. Both types are common causes of ischemic manifestation, intracranial hemorrhage, and cognitive impairment. Although both types can be associated with similar imaging markers, including $\mathrm{CMB}, \mathrm{WMH}$, lacunar infarcts, and PVS, the anatomic distribution of these markers can be helpful to differentiate these 2 entities radiologically. Lobar/superficial and cortical distribution is consistently associated with CAA, whereas the involvement of deep brain regions and the basal ganglia is most often associated with $\mathrm{HA}$ (Fig 4 and Table 2). ${ }^{15,33,36,42-44}$ Furthermore, deep CMB are typically associated with arteriolosclerosis, whereas a strictly superficial/lobar location is characteristic of CAA. ${ }^{10} \mathrm{CAA}$ is characteristically associated with lobar CMV, cSS, centrum semiovale perivascular spaces, and multiple punctate FLAIR WMH (typically $>10$ ) with a predilection for the posterior regions. ${ }^{15,33,44}$ A peri-basal ganglia pattern of WMH is strongly linked to arteriolosclerosis (Fig 4). ${ }^{15}$

\section{Clinical Implications}

Clinical manifestations of SVD and poor brain health can be varied. The presenting clinical symptoms can be abrupt, such as a 


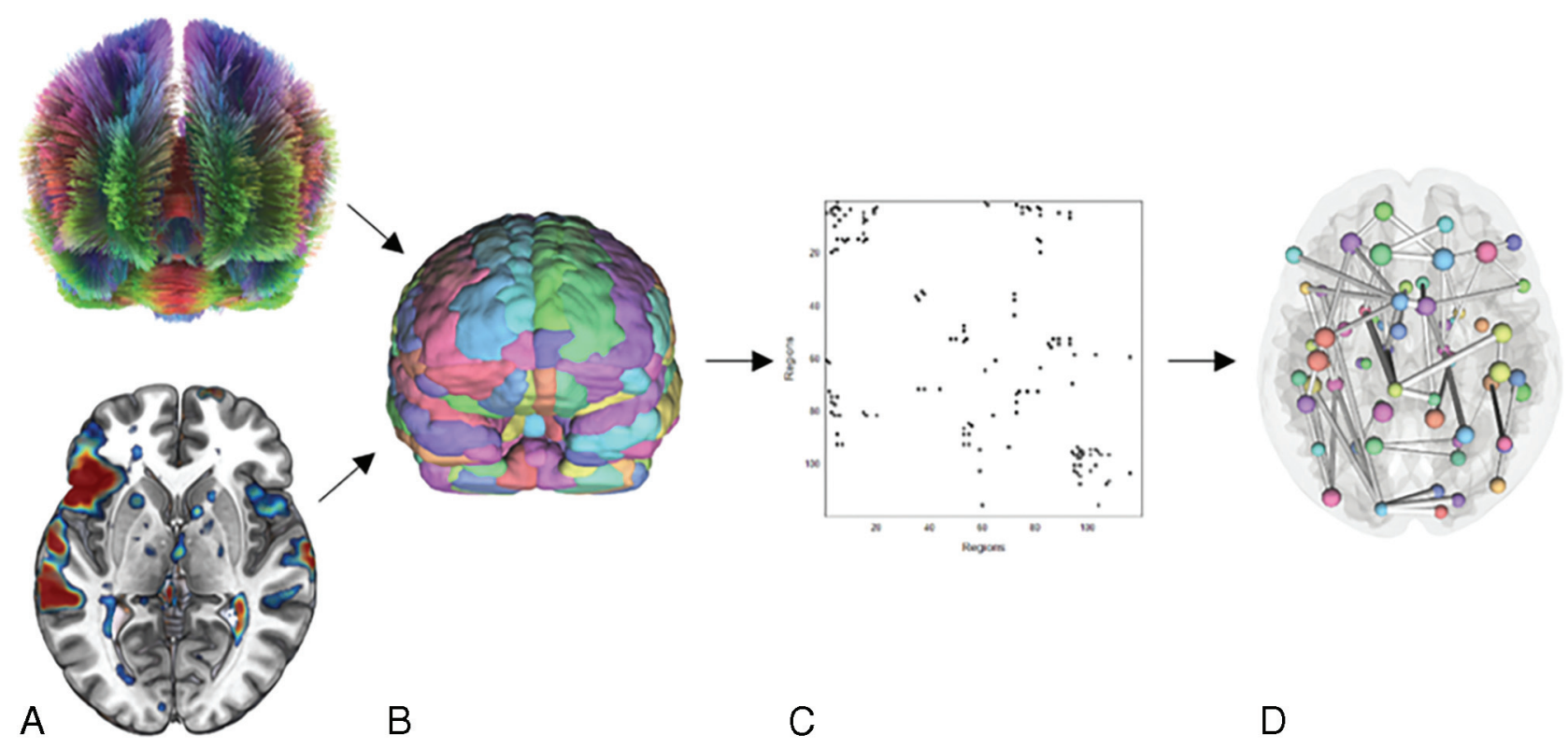

FIG 6. Sample pipeline for studying brain connectivity for assessing global brain health. A, Data are processed to generate structural (tractography) or functional (fMRI) connectivity measures. B, A parcellation scheme is applied to obtain connectivity between different regions. $C$, The connectivity data are represented as an adjacency matrix. $D$, Network properties are calculated on the adjacency matrix. In the sample image, regions in the network are sized by importance (ie, centrality) and edges are sized by the strength of the connection.

resulting intracerebral hemorrhage $(\mathrm{ICH})$ or, more insidious, manifesting as progressive cognitive decline, mood disorders, extrapyramidal symptoms, and depression. This variability can be due to multiple factors including characteristics of vascular injury (type, location, and extent), the degree of comorbidities, and resilience factors such as brain reserve, which refers to the capacity to cope with brain pathology. ${ }^{5}$ Many clinical symptoms relate to secondary neurodegeneration due to the global effects of SVD such as brain atrophy. ${ }^{5,45-50}$ Several studies have shown that subcortical infarcts and WMH can induce loss of a connected cortex distal to the infarct through degeneration of white matter tracts, which results in secondary cortical thinning, and neurodegeneration. ${ }^{45,47,48}$ The main clinical manifestations of SVD follow.

SVD and Ischemic Stroke. SVD causes approximately 25\% of all acute ischemic strokes, mainly in the form of lacunar infarction. ${ }^{10}$ Although stroke recurrence is similar to that in other stroke subtypes, the proportion of patients with lacunar infarct who are dependent at 3 years is $42 \% .{ }^{10}$ Approximately $30 \%$ of patients with lacunar stroke will develop cognitive impairment, mostly involving executive functions, attention, and psychomotor speed. ${ }^{10}$

SVD and ICH. ICH presumed due to SVD can result in devastating complications with high morbidity and mortality and a significant risk of recurrence. ${ }^{51} \mathrm{CMB}$ are present in $52 \%$ of patients with first-ever ICH and $83 \%$ of those with recurrent $\mathrm{ICH} .{ }^{51}$ The location of recurrent $\mathrm{ICH}$ can relate to the distribution and severity of underlying SVD, particularly CMB and superficial siderosis, which could help in identifying patients at high risk of ICH recurrence. ${ }^{51}$ Furthermore, it is reported that the presence of $\geq 5 \mathrm{CMB}$ can be associated with recurrent lobar $\mathrm{ICH}^{33}$
SVD and Cognitive Decline. SVD coexists with Alzheimer disease and Alzheimer disease-related dementia, can worsen cognitive outcome, and is the most common cause of vascular dementia, contributing to about $50 \%$ of dementias. ${ }^{5-7,10}$ Recent data suggest that WMH burden is associated with an increased risk of developing dementia, including the Alzheimer disease type, ${ }^{3}$ and that arteriolosclerosis increases the odds of Alzheimer disease dementia, independent of the effect of Alzheimer disease pathology. ${ }^{10}$

SVD and Parkinsonism. SVD at baseline, particularly WMH burden and lacunes, was associated with an increased risk of parkinsonism. ${ }^{52}$ Furthermore, microbleeds and gray matter atrophy were found to increase the risk of vascular parkinsonism. ${ }^{47}$

\section{Advanced Imaging for SVD}

While SVD markers are extremely common findings on conventional MR imaging, there are still weak associations of the imaging markers and the heterogeneous clinical expression of SVD. ${ }^{8,53}$ One potential reason is that conventional MR imaging measures tend to be focal, whereas SVD is increasingly thought of as a global, wholebrain phenomenon. Furthermore, the severity and nature of clinical symptoms can be vastly different in patients with radiologically identical SVD lesions. ${ }^{47}$ A network-based and/or global approach to observe subclinical SVD manifestations may explain this discrepancy. Advanced imaging techniques such as high-field MR imaging, DTI, blood oxygen level-dependent (BOLD) MR imaging, and perfusion imaging show considerable promise. ${ }^{2}$

High-Field MR Imaging. A recent MR imaging-identifiable marker of SVD is cortical microinfarcts. Microinfarcts, especially those in the deep gray matter, are best detected with an MR imaging field strength of $\geq 3 \mathrm{~T}$. High-field (7T) MR imaging shows a greater ability to detect remote microinfarcts. ${ }^{54}$ Most interesting, postmortem 
studies have shown that the penumbra of microinfarcts can extend up to 12 times larger than the microinfarct core, which is the only component visible with conventional MR imaging. ${ }^{47}$ Characterizing the penumbra and distribution of microinfarcts may be clinically important because they are known to lead to a reduced capability of adjacent axons to conduct action potentials. ${ }^{55}$

DTI. In addition to measures related to white matter integrity, such as fractional anisotropy, one can also characterize the structural connectivity with tractography, an important marker for global brain health and cognition (Fig 6). In connectivity space, regions are often called "nodes" and the connections between them are called "edges." The collection of all nodes and edges for a network can be represented as a connectivity matrix, where each row and column represent a node and entries represent connections. ${ }^{56}$ Network properties can be derived from these matrices that describe network integration and which nodes are the most important for successful functioning. Studies using these network measures have shown that patients with SVD have decreased local and global network integration in the prefrontal and interhemispheric tracts, compared with healthy controls. ${ }^{57}$ Additionally, studies have suggested that SVD may lead to loss of energy-dependent long-range white matter connections, which disrupt the structure of the network. This can result in lowered efficiency and connectivity of the most important regions in the brain for normal functioning (sometimes called the "rich-club"), leading to impaired cognition. ${ }^{58}$ The connectivity and whole brain-based studies are gaining popularity and may lead to the discovery of novel biomarkers and explanations for the heterogeneity of symptoms seen in SVD.

BOLD MRI. BOLD MR imaging is a technique that measures cerebral blood flow by detecting relative regional differences in oxygenated and deoxygenated blood supply. ${ }^{59}$ Cerebrovascular reactivity can be derived using this technique in tandem with induced hypercapnia (called a $\mathrm{CO}_{2}$ challenge). A recent study in patients with SVD showed that lower cerebrovascular reactivity, increased venous pulsatility, and decreased foramen magnum CSF volume were associated with a greater burden of WMH and a more severe basal ganglia rating of PVS. ${ }^{60}$ Other studies have indicated the role of aortic arch stiffening as a potential contributor in SVD through excessive pulsatile energy transmitted to the cerebral vasculature as part of brain-heart coupling. ${ }^{61}$

Another use of BOLD MR imaging is fMRI. Like tractography, fMRI can be analyzed in terms of brain connectivity. Using connectivity-based techniques with resting-state fMRI data, researchers have shown that patients with SVD have reduced connectivity in the prefrontal, parietal, and cingulate cortices, with corresponding increases in cerebellar connectivity and impaired deactivation of the default mode network. ${ }^{62,63}$

Perfusion Imaging. Recently, compromised BBB integrity has gained traction as an early biomarker for SVD and may play an important role in understanding SVD pathogenesis. ${ }^{64}$ There are 2 primary methods for measuring BBB integrity and microvasculature with MR imaging. The first, and most reliable method, is dynamic contrast-enhanced MR imaging with the use of an appropriate pharmacokinetic model. ${ }^{65}$ Using this technique, researchers have shown a positive correlation of SVD severity (ie, worse $\mathrm{SVD}=$ more compromised $\mathrm{BBB}$ ) and decreased BBB integrity in patients with lacunar stroke and worse clinical outcome. ${ }^{66,67}$ The second method, which does not involve a contrast agent, is arterial spin-labeling. ${ }^{68}$ While arterial spin-labeling has not been used to detect early biomarkers of SVD, it has been used to differentiate subgroups of patients with SVD. For example, a recent study showed that $\mathrm{CBF}$, measured by $3 \mathrm{D}$ arterial spin-labeling, was significantly decreased globally and locally in the temporal lobe, frontal lobe, hippocampus, thalamus, and insula in patients with increasing subcortical vascular cognitive impairment. ${ }^{69}$ There is a rich research opportunity to fully characterize the properties of the microvasculature in patients with SVD using arterial spin-labeling.

The multitude of advanced imaging techniques being used now and yet to be developed will undoubtedly lead to a redefinition of how we understand and think about SVD.

\section{Vessel Wall Imaging}

MRA and CTA are important to detect abnormalities in the lumen of vessels but cannot characterize the vessel wall. ${ }^{70}$ Vessel wall imaging has recently been developed to address this limitation, enabling better detection of nonstenotic lesions and better characterization of stenotic lesions. ${ }^{71,72}$ Briefly, vessel wall imaging is achieved using a sequence with high spatial resolution with optimal contrast-to-noise, attained by suppressing luminal blood. ${ }^{71}$ Several parameters affect the quality of the resulting images, such as field strength, 2D-versus-3D imaging, and a blood-nulling technique, but there has not yet been a thorough analysis of the tradeoffs among these permutations. ${ }^{71}$ Intracranial atherosclerosis provides a key example of how vessel wall imaging can advance our knowledge of SVD. Intracranial atherosclerosis has been downplayed as a contributor to SVD, likely because traditional vascular sequences (ie, MRA) can only assess luminal stenosis in large arteries. ${ }^{73}$ Vessel wall imaging has revealed that intracranial atherosclerosis burden, which is dependent on features of both the lumen and the vessel wall, is related to cortical and subcortical infarcts, microinfarcts, and $\mathrm{WMH}^{73}$ Several other pathologies can be captured by vessel wall imaging and may elucidate the etiology of SVD, such as atherosclerotic plaque, large-vessel vasculitis, and discrimination of reversible cerebral vasoconstriction syndrome. ${ }^{70}$

\section{Artificial Intelligence Advances in SVD}

While identifying the presence of at least 1 lacune or cerebral microbleed is straightforward in most situations, visual grading of $\mathrm{WMH}$ and PVS poses a unique challenge because the rating scales are inherently subjective, with poor agreement among neuroradiologists. ${ }^{74}$ MR imaging, specifically T2 FLAIR imaging, is the primary way that $\mathrm{WMH}$ are assessed due to even higher interreader variability using CT scans. An automated algorithm using CT scans was able to perform as well as experts at delineating WM lesions, but it varied widely with $>90 \%$ around the mean estimate. ${ }^{75}$ Previous studies have shown some success in predicting the evolution of WMH and cognitive outcomes in patients with $\mathrm{WMH}^{76,77}$ However, more research is needed on the quantification of WMH because the studies with this focus have been largely in patients with multiple sclerosis. ${ }^{78,79} \mathrm{WMH}$ in patients with additional findings, such as lacunar or cortical infarcts, 
present a difficult challenge for current algorithms. Furthermore, fully automated quantification of WMH, either by the Fazekas scale or volumetric measurement using a standard of care medical imaging (rather than research sequences) is difficult. Attempting to grade PVS with automated artificial intelligence tools is even more of a challenge, with only 1 study that showed good performance in the centrum semiovale but poor performance in the basal ganglia. ${ }^{80}$ There are great opportunities for future research to explore these rating scales for greater intersubject agreement, particularly in routine imaging, and come up with efficient and reproducible artificial intelligence brain health solutions.

\section{CONCLUSIONS AND FUTURE DIRECTIONS}

Small vessel disease is a rising epidemic associated with detrimental brain health. Neuroimaging plays a fundamental role in identifying SVD. The recognition of endothelial and neurogliovascular unit dysfunction as the main underlying mechanisms of SVD is fundamental to develop the role of advanced neuroimaging techniques. A more comprehensive approach to gauge total SVD burden, rather than individualizing and classifying each of these markers separately, is needed in future studies. This may provide a more complete estimate of the full impact of SVD on the brain to better assess total brain health and the effect of SVD-related brain damage. Finally, as radiologists, it is critical that we use standardized terminology to describe SVD in both clinical and research settings.

Disclosures: Pooja Khatri-RELATED: Grant: National Institutes of Health.* UNRELATED: Consultancy: Lumosa Therapeutics, Diamedica Therapeutics*; Grants/ Grants Pending: Cerenovus*; Royalties: UpToDate; Other: Bayer, Basking Biosciences, Comments: Bayer, National Trial Leader; Basking Biosciences, Scientific Advisory Board. Brett Kissela-RELATED: Grant: National Institutes of Health/ National Institute of Neurological Disorders and Stroke.* Rhonna Shatz-RELATED: Grant: National Institutes of Health.* Achala Vagal—RELATED: Grant: National Institutes of Health, Cerenovus,* Comments: R01, National Institutes of Health/ National Institute of Neurological Disorders and Stroke, ${ }^{\star}$ NS103824; RF, National Institute of Neurological Disorders and Stroke/National Institute on Aging, NS117643; R01, National Institutes of Health/National Institute of Neurological Disorders and Stroke, NS100417; National Institutes of Health/National Institute of Neurological Disorders and Stroke, IU01NS100699; National Institutes of Health/ National Institute of Neurological Disorders and Stroke, U01NS110772; Principal Investigator, Imaging Core Lab, ENDOLOW Trial, Cerenovus. *Money paid to the institution.

\section{REFERENCES}

1. Pantoni L. Cerebral small vessel disease: from pathogenesis and clinical characteristics to therapeutic challenges. Lancet Neurol 2010;9:689-701 CrossRef Medline

2. Wardlaw JM, Smith C, Dichgans M. Mechanisms of sporadic cerebral small vessel disease: insights from neuroimaging. Lancet Neurol 2013;12:483-97 CrossRef Medline

3. Debette S, Schilling S, Duperron MG, et al. Clinical significance of magnetic resonance imaging markers of vascular brain injury: a systematic review and meta-analysis. JAMA Neurol 2019;76:81-94 CrossRef Medline

4. Georgakis MK, Duering M, Wardlaw JM, et al. WMH and long-term outcomes in ischemic stroke: a systematic review and meta-analysis. Neurology 2019;92:e1298-1308 CrossRef Medline

5. Wardlaw JM, Smith C, Dichgans M. Small vessel disease: mechanisms and clinical implications. Lancet Neurol 2019;18:684-96 CrossRef Medline
6. Kapasi A, DeCarli C, Schneider JA. Impact of multiple pathologies on the threshold for clinically overt dementia. Acta Neuropathol 2017;134:171-86 CrossRef Medline

7. Bos D, Wolters FJ, Darweesh SKL, et al. Cerebral small vessel disease and the risk of dementia: a systematic review and meta-analysis of population-based evidence. Alzheimers Dement 2018;14:1482-12 CrossRef Medline

8. Shibuya M, da Costa Leite C, Lucato LT. Neuroimaging in cerebral small vessel disease: update and new concepts. Dement Neuropsychol 2017;11:336-42 CrossRef Medline

9. Gorelick PB, Furie KL, Iadecola C, et al. Defining optimal brain health in adults. Stroke 2017;48:e284-303 CrossRef Medline

10. Pasi M, Cordonnier C. Clinical relevance of cerebral small vessel diseases. Stroke 2020;51:47-53 CrossRef Medline

11. Wardlaw JM, Smith EE, Biessels GJ, et al; STandards for ReportIng Vascular changes on nEuroimaging (STRIVE v1). Neuroimaging standards for research into small vessel disease and its contribution to ageing and neurodegeneration. Lancet Neurol 2013;12:82238 CrossRef Medline

12. van Veluw SJ, Shih AY, Smith EE, et al. Detection, risk factors, and functional consequences of cerebral microinfarcts. Lancet Neurol 2017;16:730-40 CrossRef Medline

13. Potter GM, Marlborough FJ, Wardlaw JM. Wide variation in definition, detection, and description of lacunar lesions on imaging. Stroke 2011;42:359-66 CrossRef Medline

14. Zhu YC, Dufouil C, Tzourio C, et al. Silent brain infarcts. Stroke 2011;42:1140- 45 CrossRef Medline

15. Charidimou A, Boulouis G, Haley K, et al. White matter hyperintensity patterns in cerebral amyloid angiopathy and hypertensive arteriopathy. Neurology 2016;86:505-11 CrossRef Medline

16. Fisher CM. Lacunar infarcts: a review. Cerebravascular Diseases 1991;1:311-20 CrossRef

17. Fisher CM. Lacunar strokes and infarcts. Neurology 1982;32:871-76 CrossRef CrossRef Medline

18. Bailey EL, Smith C, Sudlow CL, et al. Pathology of lacunar ischemic stroke in humans: a systematic review. Brain Pathol 2012;22:583-91 CrossRef Medline

19. Kim JS, Yoon Y. Single subcortical infarction associated with parental arterial disease: important yet neglected sub-type of atherothrombotic stroke. Int J Stroke 2013;8:197-203 CrossRef Medline

20. Shi Y, Wardlaw JM. Update on cerebral small vessel disease: a dynamic whole-brain disease. Stroke Vasc Neurol 2016;1:83-92 CrossRef Medline

21. Weber R, Weimar C, Blatchford J, et al; PRoFESS Imaging Substudy Group. Telmisartan on top of antihypertensive treatment does not prevent progression of cerebral white matter lesions in the Prevention Regimen for Effectively Avoiding Second Strokes (PRoFESS) MRI substudy. Stroke 2012;43:2336-42 CrossRef Medline

22. Jackson CA, Hutchinson A, Dennis MS, et al. Differing risk factor profiles of ischemic stroke subtypes: evidence for a distinct lacunar arteriopathy? Stroke 2010;41:624-29 CrossRef Medline

23. Lammie GA, Brannan F, Slattery J, et al. Nonhypertensive cerebral small-vessel disease. Stroke 1997;28:2222-29 CrossRef Medline

24. Godin O, Tzourio C, Maillard P, et al. Antihypertensive treatment and change in blood pressure are associated with the progression of white matter lesion volumes. Circulation 2011;123:266-73 CrossRef Medline

25. Bosetti F, Galis ZS, Bynoe MS, et al; "Small Blood Vessels: Big Health Problems" Workshop Participants. Small blood vessels: big health problems?": scientific recommendations of the National Institutes of Health Workshop. J Am Heart Assoc 2016;5:e043898 CrossRef Medline

26. Petersen MA, Ryu JK, Akassoglou K. Fibrinogen in neurological diseases: mechanisms, imaging and therapeutics. Nat Rev Neurosci 2018;19:283-301 CrossRef Medline 
27. Wuerfel J, Haertle M, Waiczies $\mathrm{H}$, et al. Perivascular spaces: MRI marker of inflammatory activity in the brain? Brain 2008;131:233240 CrossRef Medline

28. Hawkins BT, Davis TP. The blood-brain barrier/neurovascular unit in health and disease. Pharmacol Rev 2005;57:173-85 CrossRef Medline

29. Bechmann I, Galea I, Perry VH. What is the blood-brain barrier (not)? Trends Immunol 2007;28:5-11 CrossRef Medline

30. Iliff JJ, Wang M, Zeppenfeld DM, et al. Cerebral arterial pulsation drives paravascular CSF-interstitial fluid exchange in the murine brain. J Neurosci 2013;33:18190-99 CrossRef Medline

31. Caunca MR, De Leon-Benedetti A, Latour L, et al. Neuroimaging of cerebral small vessel disease and age-related cognitive changes. Front Aging Neurosci 2019;11:145 CrossRef Medline

32. Das AS, Regenhardt RW, Vernooij MW, et al. Asymptomatic cerebral small vessel disease: insights from population-based studies. $J$ Stroke 2019;21:121-38 CrossRef Medline

33. Charidimou A, Martinez-Ramirez S, Reijmer YD, et al. Total magnetic resonance imaging burden of small vessel disease in cerebral amyloid angiopathy: an imaging-pathologic study of concept validation. JAMA Neurol 2016;73:994-1001 CrossRef Medline

34. Fazekas F, Chawluk J, Alavi A, et al. MR signal abnormalities at $\mathbf{1 . 5}$ T in Alzheimer's dementia and normal aging. AJR Am J Roentgenol 1987;149:351-56 CrossRef Medline

35. Zhu YC, Chabriat H, Godin O, et al. Distribution of white matter hyperintensity in cerebral hemorrhage and healthy aging. J Neurol 2012;259:530-36 CrossRef Medline

36. Passiak BS, Liu D, Kresge HA, et al. Perivascular spaces contribute to cognition beyond other small vessel disease markers. Neurology 2019;92:e1309-21 CrossRef Medline

37. Gregoire SM, Chaudhary UJ, Brown MM, et al. The Microbleed Anatomical Rating Scale (MARS). Neurology 2009;73:1759-66 CrossRef Medline

38. Cordonnier C, Potter GM, Jackson CA, et al. Improving interrater agreement about brain microbleeds. Stroke 2009;40:94-99 CrossRef Medline

39. Dou Q, Chen H, Yu L, et al. Automatic detection of cerebral microbleeds from MR images via 3D convolutional neural networks. IEEE Trans Med Imaging 2016;35:1182-95 CrossRef Medline

40. Staals J, Makin SD, Doubal FN, et al. Stroke subtype, vascular risk factors, and total MRI brain small-vessel disease burden. Neurology 2014;83:1228-34 CrossRef Medline

41. Banerjee G, Jang H, Kim HJ, et al. Total MRI small vessel disease burden correlates with cognitive performance, cortical atrophy, and network measures in a memory clinic population. J Alzheimers Dis 2018;63:1485-97 CrossRef Medline

42. Klarenbeek P, van Oostenbrugge RJ, Rouhl RPW, et al. Ambulatory blood pressure in patients with lacunar stroke. Stroke 2013;44:2995-99 CrossRef Medline

43. Pasi M, Boulouis G, Fotiadis P, et al. Distribution of lacunes in cerebral amyloid angiopathy and hypertensive small vessel disease. Neurology 2017;88:2162-68 CrossRef Medline

44. Shams S, Martola J, Charidimou A, et al. Topography and determinants of magnetic resonance imaging (MRI)-visible perivascular spaces in a large memory clinic cohort. J Am Heart Assoc 2017;6: e006229 CrossRef Medline

45. Duering M, Righart R, Wollenweber FA, et al. Acute infarcts cause focal thinning in remote cortex via degeneration of connecting fiber tracts. Neurology 2015;84:1685-92 CrossRef

46. Dickie DA, Karama S, Ritchie SJ, et al. Progression of white matter disease and cortical thinning are not related in older communitydwelling subjects. Stroke 2016;47:410-16 CrossRef Medline

47. Ter Telgte A, van Leijsen EM, Wiegertjes $\mathrm{K}$, et al. Cerebral small vessel disease: from a focal to a global perspective. Nat Rev Neurol 2018;14:387-98 CrossRef Medline

48. Peres R, De Guio F, Chabriat H, et al. Alterations of the cerebral cortex in sporadic small vessel disease: a systematic review of in vivo MRI data. J Cereb Blood Flow Metab 2016;36:681-95 CrossRef Medline
49. Chabriat H, Hervé D, Duering M, et al. Predictors of clinical worsening in cerebral autosomal dominant arteriopathy with subcortical infarcts and leukoencephalopathy. Stroke 2016;47:4-11 CrossRef Medline

50. Rizvi B, Narkhede A, Last BS, et al. The effect of white matter hyperintensities on cognition is mediated by cortical atrophy. Neurobiol Aging 2018;64:25-32 CrossRef Medline

51. Charidimou A, Imaizumi T, Moulin S, et al. Brain hemorrhage recurrence, small vessel disease type, and cerebral microbleeds: a meta-analysis. Neurology 2017;89:820-29 CrossRef Medline

52. van der Holst HM, De Leeuw FE. Author response. Neurology 2016;86:1268-69 CrossRef Medline

53. Gouw AA, Seewann A, van der Flier WM, et al. Heterogeneity of small vessel disease: a systematic review of MRI and histopathology correlations. J Neurol Neurosurg Psychiatry 2011;82:126-35 CrossRef Medline

54. van Rooden S, Goos JD, van Opstal AM, et al. Increased number of microinfarcts in Alzheimer disease at 7-T MR imaging. Radiology 2014;270:205-11 CrossRef

55. Coban H, Tung S, Yoo B, et al. Molecular disorganization of axons adjacent to human cortical microinfarcts. Front Neurol 2017;8:405 CrossRef Medline

56. Rubinov M, Sporns O. Complex network measures of brain connectivity: uses and interpretations. Neuroimage 2010;52:1059-69 CrossRef Medline

57. Lawrence AJ, Chung AW, Morris RG, et al. Structural network efficiency is associated with cognitive impairment in small-vessel disease. Neurology 2014;83:304-11 CrossRef Medline

58. Marebwa BK, Adams RJ, Magwood GS, et al. Cardiovascular risk factors and brain health: impact on long-range cortical connections and cognitive performance. J Am Heart Assoc 2018;7:e010054 CrossRef Medline

59. Ogawa S, Tank DW, Menon R, et al. Intrinsic signal changes accompanying sensory stimulation: functional brain mapping with magnetic resonance imaging. Proc Natl Acad Sci U S A 1992;89:5951-55 CrossRef Medline

60. Blair GW, Thrippleton MJ, Shi Y, et al. Intracranial hemodynamic relationships in patients with cerebral small vessel disease. Neurology 2020;94:e2258-69 CrossRef Medline

61. Aghilinejad A, Amlani F, King KS, et al. Dynamic effects of aortic arch stiffening on pulsatile energy transmission to cerebral vasculature as a determinant of brain-heart coupling. Sci Rep 2020;10:8784 CrossRef Medline

62. Papma JM, den Heijer T, de Koning I, et al. The influence of cerebral small vessel disease on default mode network deactivation in mild cognitive impairment. Neuroimage Clin 2012;2:33-42 CrossRef Medline

63. Schaefer A, Quinque EM, Kipping JA, et al. Early small vessel disease affects frontoparietal and cerebellar hubs in close correlation with clinical symptoms: a resting-state fMRI study. J Cereb Blood Flow Metab 2014;34:1091-95 CrossRef Medline

64. Li Y, Li M, Zuo L, et al. Compromised blood-brain barrier integrity is associated with total magnetic resonance imaging burden of cerebral small vessel disease. Front Neurol 2018;9:221 CrossRef Medline

65. Heye AK, Thrippleton MJ, Armitage PA, et al. Tracer kinetic modelling for DCE-MRI quantification of subtle blood-brain barrier permeability. Neuroimage 2016;125:446-55 CrossRef Medline

66. Topakian R, Barrick TR, Howe FA, et al. Blood-brain barrier permeability is increased in normal-appearing white matter in patients with lacunar stroke and leukoaraiosis. J Neurol Neurosurg Psychiatry 2010;81:192-97 CrossRef Medline

67. Wardlaw JM, Doubal FN, Valdes-Hernandez M, et al. Blood-brain barrier permeability and long-term clinical and imaging outcomes in cerebral small vessel disease. Stroke 2013;44:525-27 CrossRef Medline

68. Hendrikse J, Petersen ET, Golay X. Vascular disorders: insight from arterial spin-labeling. Neuroimaging Clin N Am 2012;22:25969 CrossRef Medline 
69. Sun Y, Cao W, Ding W, et al. Cerebral blood flow alterations as assessed by 3D ASL in cognitive impairment in patients with subcortical vascular cognitive impairment: a marker for disease severity. Front Aging Neurosci 2016;8:211 CrossRef Medline

70. Mandell DM, Mossa-Basha M, Qiao Y, et al; Vessel Wall Imaging Study Group of the American Society of Neuroradiology. Intracranial vessel wall MRI: Principles and Expert Consensus Recommendations of the American Society of Neuroradiology. AJNR Am J Neuroradiol 2017;38:218-29 CrossRef Medline

71. Lindenholz A, van der Kolk AG, Zwanenburg JJM, et al. The use and pitfalls of intracranial vessel wall imaging: how we do it. Radiology 2018;286:12-28 CrossRef Medline

72. Farag S, El-Dien HZ, Abdelazeem Y, et al. Value of vessel wall magnetic resonance imaging in the diagnosis of cerebrovascular diseases. Egypt J Neurol Psychiatry Neurosurg 2020;56:114 CrossRef

73. Zwartbol MH, van der Kolk AG, Kuijf HJ, et al; UCC-SMART Study Group. Intracranial vessel wall lesions on 7T MRI and MRI features of cerebral small vessel disease: the SMART-MR study. $J$ Cereb Blood Flow Metab 2021;41:1219-28 CrossRef Medline

74. Mårtensson G, Ferreira D, Cavallin L, et al; Alzheimer's Disease Neuroimaging Initiative. AVRA: Automatic visual ratings of atrophy from MRI images using recurrent convolutional neural networks. Neuroimage Clin 2019;23:101872 CrossRef Medline
75. Chen L, Carlton Jones AL, Mair G, et al; IST-3 Collaborative Group. Rapid automated quantification of cerebral leukoaraiosis on CT images: a multicenter validation study. Radiology 2018;288:573-81 CrossRef Medline

76. Rachmadi MF, Valdés-Hernández $\mathrm{M}$ del $\mathrm{C}$, Makin S, et al. Automatic spatial estimation of white matter hyperintensities evolution in brain MRI using disease evolution predictor deep neural networks. April 21, 2020. bioRxiv. https://www.biorxiv.org/content/ 10.1101/738641v3. Accessed April 21, 2020

77. Schirmer MD, Dalca AV, Sridharan R, et al. White matter hyperintensity quantification in large-scale clinical acute ischemic stroke cohorts: the MRI-GENIE study. Neuroimage Clin 2019;23:101884 CrossRef Medline

78. Jiang J, Liu T, Zhu W, et al. UBO Detector: a cluster-based, fully automated pipeline for extracting white matter hyperintensities. Neuroimage 2018;174:539-49 CrossRef Medline

79. Schmidt P, Pongratz V, Küster P, et al. Automated segmentation of changes in FLAIR-hyperintense white matter lesions in multiple sclerosis on serial magnetic resonance imaging. Neuroimage Clin 2019;23:101849 CrossRef Medline

80. Dubost F, Yilmaz P, Adams H, et al. Enlarged perivascular spaces in brain MRI: Automated quantification in four regions. Neuroimage 2019;185:534-44 CrossRef Medline 